\title{
THE NORMAL APPROXIMATION TO THE POISSON DISTRIBUTION AND A PROOF OF A CONJECTURE OF RAMANUJAN ${ }^{1}$
}

TSENG TUNG CHENG

1. Summary. The Poisson distribution with parameter $\lambda$ is given by

$$
F(x)=\sum_{r=0}^{n} p_{r} \quad \text { where } \quad p_{r}=\frac{\lambda^{r}}{r !} e^{-\lambda}, \quad n=[x],
$$

for $x \geqq 0$. It is well known that $F(x)$ converges to the normal distribution as $\lambda \rightarrow \infty$. We shall prove the following theorem.

THEOREM I. Let $x=\lambda^{-1 / 2}(n-\lambda+1 / 2)$. Then

$$
\begin{aligned}
\sum_{r=0}^{n} \frac{\lambda^{r}}{r !} e^{-\lambda}=(2 \pi)^{-1 / 2} \int_{-\infty}^{x} e^{-(1 / 2) t^{2}} d t & \\
& +(1 / 6)(2 \pi \lambda)^{-1 / 2}\left(1-x^{2}\right) e^{-(1 / 2) x^{2}}+\delta,
\end{aligned}
$$

where $\delta$ satisfies the inequality

$$
|\delta|<.076 \lambda^{-1}+.043 \lambda^{-3 / 2}+.13 \lambda^{-2} .
$$

This formula is analogous to Uspensky's [1 $]^{2}$ estimate of the error term in the normal approximation to the binormal distribution and our proof consists in an adaptation of Uspensky's method.

At the same time we verify the following conjecture of Ramanujan: for every positive integer $n$ the value of $\theta$ which satisfies the equation

$$
\left\{1+\frac{n}{1 !}+\cdots+\frac{n^{n-1}}{(n-1) !}+\theta \frac{n^{n}}{n !}\right\} e^{-n}=1 / 2
$$

lies between $1 / 2$ and $1 / 3$, and tends to $1 / 3$ as $n \rightarrow \infty$. A proof of the above statement was given by Szegö [3]. We give a more elementary proof, using only standard tools, for the following theorem.

Theorem II. For $n \geqq 7$ the root of the equation (1.4) in $\theta$ lies between .37 and $1 / 3$.

Finally, we shall obtain the following asymptotic expansion of $\theta$ :

Received by the editors March 15, 1948.

1 This paper contains the results of a master's thesis done under the direction of Professor W. Feller and accepted by Cornell University in September, 1947.

2 Numbers in brackets refer to the references cited at the end of the paper. 


$$
\theta=1 / 3+(4 / 135) n^{-1}+(8 / 2835) n^{-2}+O\left(n^{-3}\right) .
$$

2. Proof of Theorem I. From the characteristic function

$$
\sum_{r=0}^{\infty} p_{r} e^{i r t}=e^{\lambda(e i t-1)}
$$

we get

$$
\int_{-\pi}^{\pi} e^{-i r t} \cdot e^{\lambda\left(e^{i t-1)}\right.} d t=2 \pi p_{r}
$$

From (2.1) it follows that

$$
\begin{aligned}
\sum_{r=0}^{n} p_{r}= & (2 \pi)^{-1} \int_{0}^{\pi}(\sin t / 2)^{-1} \\
& \cdot \exp \{\lambda(\cos t-1)\} \sin [(n+1 / 2) t-\lambda \sin t] d t \\
& +(2 \pi)^{-1} \int_{0}^{\pi}(\sin t / 2)^{-1} \exp \{\lambda(\cos t-1)\} \sin (\lambda \sin t+t / 2) d t
\end{aligned}
$$

The second integral is independent of $\lambda$, since its derivative with respect to $\lambda$ vanishes, as may easily be verified. Putting $\lambda=0$ we see the value of the integral is $1 / 2$. Hence we have

$$
\begin{aligned}
\sum_{r=0}^{n} \frac{\lambda^{r}}{r !} e^{-\lambda}-\frac{1}{2} & =(2 \pi)^{-1} \int_{0}^{\pi}(\sin t / 2)^{-1} \\
& \cdot \exp \{\lambda(\cos t-1)\} \sin [(n+1 / 2) t-\lambda \sin t] d t \\
= & I
\end{aligned}
$$

To estimate the integral $I$ we introduce

$$
I_{1}=(2 \pi)^{-1} \int_{0}^{\infty} e^{-\lambda t^{2} / 2}(t / 2)^{-1} \sin \left[\lambda^{1 / 2} x t+\lambda(t-\sin t)\right] d t
$$

Then

$$
\begin{aligned}
\left|I-I_{1}\right| \leqq & (2 \pi)^{-1} \int_{\pi}^{\infty} e^{-\lambda t^{2} / 2}(t / 2)^{-1} d t \\
& +(2 \pi)^{-1} \int_{0}^{\pi}\left\{e^{-2 \lambda \sin ^{2} t / 2}(\sin t / 2)^{-1}-e^{-\lambda t^{2} / 2}(t / 2)^{-1}\right\} d t \\
& =J_{1}+J_{2}+J_{3}
\end{aligned}
$$

where the integrals $J_{k}$ are defined and estimated as follows: 


$$
\begin{aligned}
J_{1} & =(2 \pi)^{-1} \int_{0}^{\pi}\left\{e^{-2 \lambda \sin ^{2} t / 2}(\sin t / 2)^{-1} \cos t / 2-e^{-\lambda t^{2} / 2}(t / 2)^{-1}\right\} d t \\
& =-(2 \pi)^{-1} \int_{2}^{\pi} e^{-\lambda t^{2} / 2}(t / 2)^{-1} d t<0
\end{aligned}
$$

$$
\begin{aligned}
J_{2}= & (2 \pi)^{-1} \int_{\pi}^{\infty} e^{-\lambda t^{2} / 2}(t / 2)^{-1} d t<(32 \pi)^{-1} \int_{\pi}^{\infty} t^{3} e^{-2 \lambda t^{2} / \pi^{2}} d t \\
J_{3}= & (2 \pi)^{-1} \int_{0}^{\pi} e^{-2 \lambda \sin ^{2} t / 2}(\sin t / 2)^{-1}(1-\cos t / 2) d t \\
< & (2 \pi)^{-1} \int_{0}^{\pi} e^{-2 \lambda \sin ^{2} t / 2} \cdot(\sin t / 2)^{-1} \\
& \cdot\left\{\frac{\sin ^{2} t / 2 \cos t / 2}{2}+(1-\cos t / 2) \sin ^{2} t / 2\right\} d t \\
< & (8 \pi)^{-1} \int_{0}^{2} t e^{-\lambda t^{2} / 2} d t+(32 \pi)^{-1} \int_{0}^{\pi} t^{3} e^{-2 \lambda t^{2} / \pi^{2}} d t
\end{aligned}
$$

It follows from (2.3)-(2.6) that

$$
\begin{aligned}
\left|I-I_{1}\right| & <(8 \pi)^{-1} \int_{0}^{\infty} t e^{-\lambda t^{2} / 2} d t+(32 \pi)^{-1} \int_{0}^{\infty} t^{3} e^{-2 \lambda t^{2} / \pi^{2}} d t \\
& =(8 \pi \lambda)^{-1}+\left(\pi^{3} / 256\right) \lambda^{-2}<(8 \pi \lambda)^{-1}+.13 \lambda^{-2}
\end{aligned}
$$

Now we write

$$
\begin{aligned}
I_{1}= & \pi^{-1} \int_{0}^{\infty} t^{-1} e^{-\lambda t^{2} / 2} \cdot \sin \lambda^{1 / 2} x t \cdot \cos \lambda(t-\sin t) d t \\
& +\pi^{-1} \int_{0}^{\infty} t^{-1} e^{-\lambda t^{2} / 2} \cdot \cos \lambda^{1 / 2} x t \cdot \sin \lambda(t-\sin t) d t \\
(2.8)= & \left\{\pi^{-1} \int_{0}^{\infty} t^{-1} e^{-\lambda t^{2} / 2} \cdot \sin \lambda^{1 / 2} x t d t+J_{4}\right\} \\
& +\left\{\pi^{-1} \int_{0}^{\infty} t^{-1} e^{-\lambda t^{2} / 2} \cdot \cos \lambda^{1 / 2} x t \cdot \frac{\lambda t^{3}}{6} d t+J_{5}\right\} \\
= & (2 \pi)^{-1 / 2} \int_{0}^{x} e^{-t^{2} / 2} d t+\frac{1}{6}(2 \pi \lambda)^{-1 / 2}\left(1-x^{2}\right) e^{-x^{2} / 2}+J_{4}+J_{5},
\end{aligned}
$$

where

$$
J_{4}=\pi^{-1} \int_{0}^{\infty} t^{-1} e^{-\lambda t^{2} / 2}[\cos \lambda(t-\sin t)-1] d t,
$$




$$
J_{5}=\pi^{-1} \int_{0}^{\infty} t^{-1} e^{-\lambda t^{2} / 2} \cos \lambda^{1 / 2} x t \cdot\left[\sin \lambda(t-\sin t)-\frac{\lambda t^{3}}{6}\right] d t .
$$

Upper limits for $\left|J_{4}\right|$ and $\left|J_{5}\right|$ are obtained as follows:

$$
\left|J_{4}\right|<\pi^{-1} \int_{0}^{\infty} t^{-1} e^{-\lambda t^{2} / 2} \cdot \frac{\lambda^{2}(t-\sin t)^{2}}{2} d t
$$

$$
\begin{aligned}
< & \pi^{-1} \int_{0}^{\infty} t^{-1} e^{-\lambda t^{2} / 2} \cdot \frac{\lambda^{2} t^{6}}{72} d t=(9 \pi \lambda)^{-1} \\
\left|J_{5}\right|< & \pi^{-1} \int_{0}^{\infty} t^{-1} e^{-\lambda t^{2} / 2}\left\{\left[\frac{\lambda t^{3}}{6}-\lambda(t-\sin t)\right]\right. \\
& +[\lambda(t-\sin t)-\sin \lambda(t-\sin t)]\} d t \\
& <\pi^{-1} \int_{0}^{\infty} t^{-1} e^{-\lambda t^{2} / 2}\left[\frac{\lambda t^{5}}{120}+\frac{\lambda^{3} t^{9}}{6^{4}}\right] d t \\
= & (2 \pi)^{-1 / 2} \lambda^{-3 / 2}[1 / 40+35 / 432]<.043 \lambda^{-3 / 2}
\end{aligned}
$$

From $(2.7)-(2.10)$ and the inequality $(8 \pi)^{-1}+(9 \pi)^{-1}<.076$ the required result follows immediately.

3. Proof of Theorem II. Solving (1.4) for $\theta$ and using the formula (2.2), we get

$$
2 \theta-1=-I^{\prime} /\left(n^{n} e^{-n} / n !\right)
$$

where

$$
\begin{aligned}
I^{\prime}= & (2 \pi)^{-1} \int_{0}^{\pi}\left(\sin \frac{t}{2}\right)^{-1} \cdot \exp \{n(\cos t-1)\} \\
& \cdot\left\{\sin \left[\left(n+\frac{1}{2}\right) t-n \sin t\right]\right. \\
& \left.+\sin \left[\left(n-\frac{1}{2}\right) t-n \sin t\right]\right\} d t \\
= & \pi^{-1} \int_{0}^{\pi}\left(\sin \frac{t}{2}\right)^{-1} \cdot \exp \{n(\cos t-1)\} \cdot \cos \frac{t}{2} \cdot \sin n(t-\sin t) d t \\
= & 2 \pi^{-1} \int_{0}^{2} t^{-1} e^{-n t^{2} / 2} \cdot \sin n\left[2 \operatorname{arc} \sin \frac{t}{2}-t\left(1-\frac{t^{2}}{4}\right)^{1 / 2}\right] d t .
\end{aligned}
$$

By Maclaurin expansion,

$2 \operatorname{arc} \sin t / 2-t\left(1-t^{2} / 4\right)^{1 / 2}=t^{3} / 6+t^{5} / 80+3 t^{7} / 1792+t^{9} f(t)$, 
where $f(t)$ is an increasing function in the interval $0 \leqq t \leqq 2$. Hence $\sin n\left[2 \operatorname{arc} \sin t / 2-t\left(1-t^{2} / 4\right)^{1 / 2}\right]$

$$
\begin{aligned}
\leqq & \sin \left(n t^{3} / 6+n t^{5} / 80\right)+3 n t^{7} / 1792+n t^{9} f(2) \\
\leqq & \left(n t^{3} / 6+n t^{5} / 80\right)-\left(n t^{3} / 6+n t^{5} / 80\right)^{3} / 6 \\
& +\left(n t^{3} / 6+n t^{5} / 80\right)^{5} / 120+3 n t^{7} / 1792+.0024 n t^{9} .
\end{aligned}
$$

Assume $n \geqq 7$. Then the last expression is positive when $t>2$, and therefore

$$
\begin{gathered}
I^{\prime}<2 \pi^{-1} \int_{0}^{\infty} t^{-1} e^{-n t^{2} / 2} \cdot\left\{\frac{n t^{3}}{6}+\frac{n t^{5}}{80}-\frac{1}{6}\left(\frac{n t^{3}}{6}+\frac{n t^{5}}{80}\right)^{3}\right. \\
\left.+\frac{1}{120}\left(\frac{n t^{3}}{6}+\frac{n t^{5}}{80}\right)^{5}+\frac{3 n t^{7}}{1792}+.0024 n t^{9}\right\} d t \\
<(2 \pi n)^{-1 / 2}\left\{1 / 3-.087 n^{-1}+.012 n^{-2}+1.87 n^{-3}\right. \\
\left.+4 \cdot 1 n^{-4}+6 n^{-5}+5 n^{-6}+2 n^{-7}\right\} .
\end{gathered}
$$

In obtaining the last inequality we have, as before, used the familiar relation:

$$
2(2 \pi)^{-1 / 2} \int_{0}^{\infty} t^{2 k} e^{-(1 / 2) t^{2}} d t=1 \cdot 3 \cdots \cdot(2 k-1) .
$$

Taking in (3.2) $n \geqq 7$ we get

$$
I^{\prime}<(2 \pi n)^{-1 / 2}\left(1 / 3-.03 n^{-1}\right) .
$$

On the other hand we can write

$$
\begin{aligned}
2 \arcsin t / 2-t\left(1-t^{2} / 4\right)^{1 / 2} & =t^{3} / 6+t^{5} g(t) \\
& \geqq t^{3} / 6+.057 t^{5},
\end{aligned}
$$

because $g(t)$ increases and $g(2)<.057$. Hence

$$
\begin{aligned}
I^{\prime} & >2 \pi^{-1} \int_{0}^{2} t^{-1} e^{-(1 / 2) n t^{2}}\left\{\sin \frac{n t^{3}}{6}-.057 n t^{5}\right\} d t \\
& >2 \pi^{-1} \int_{0}^{\infty} t^{-1} e^{-(1 / 2) n t^{2}}\left\{\frac{n t^{3}}{6}-\frac{1}{6}\left(\frac{n t^{3}}{6}\right)^{3}-.057 n t^{5}\right\} d t .
\end{aligned}
$$

Therefore

$$
\left.I^{\prime}>(2 \pi n)^{-1 / 2}(1 / 3)-.505 n^{-1}\right)>.26(2 \pi n)^{-1 / 2} \text {, when } n \geqq 7 .
$$

From (3.1), (3.3), (3.4), and Stirling's formula, 


$$
(2 \pi n)^{1 / 2} n^{n} e^{-n}<n !<(2 \pi n)^{1 / 2} n^{n} e^{-n+1 / 12 n},
$$

it follows that $2 \theta-1<-.26$, or $\theta<.37$; and

$$
\begin{aligned}
2 \theta-1 & >-e^{1 / 12 n} \cdot(2 \pi n)^{1 / 2} \cdot(2 \pi n)^{-1 / 2}(1 / 3-.03 / n) \\
& >-\left(1+.09 n^{-1}\right)\left(1 / 3-.03 n^{-1}\right) \\
& =-1 / 3+.0027 n^{-2}>-1 / 3,
\end{aligned}
$$

and therefore $\theta>1 / 3$.

4. Proof of the equation (1.5). It can be verified without difficulty that

$$
I^{\prime}=(2 \pi n)^{-1 / 2}\left\{1 / 3-47 / 540 n^{-1}+71 / 6048 n^{-2}\right\}+O\left(n^{-7 / 2}\right) .
$$

From a modified form of Stirling's formula due to Feller [2], namely, for $n \geqq 4$,

$$
n !=(2 \pi n)^{1 / 2} n^{n} \exp \left\{-n+\frac{1}{12 n}-\frac{1+\delta}{360 n^{3}}\right\}, \quad|\delta|<1 / 6,
$$

it follows that

$$
\begin{aligned}
2 \theta-1= & -\left[1 / 3-47 / 540 n^{-1}+71 / 6048 n^{-2}+\cdots\right] \\
& \cdot\left[1+1 / 12 n^{-1}+1 /\left(2.12^{2}\right) n^{-2}+\cdots\right] \\
= & -1 / 3+8 / 135 n^{-1}+16 / 2835 n^{-2}+O\left(n^{-3}\right) .
\end{aligned}
$$

An obvious reduction then gives the equation (1.5).

\section{REFERENCES}

1. J. V. Uspensky, Introduction to mathematical probability, New York, 1937.

2. W. Feller, On the normal approximation to the binormal distribution, Ann. Math. Statist. vol. 16 (1945) pp. 319-329.

3. G. Szegö, Über einige von S. Ramanujan gestellte Aufgaben, J. London Math. Soc. vol. 3 (1928) pp. 225-232.

CORNELL UNIVERSITY 